\title{
أسرار العقل الصهيوني
}

المؤلف: الدكتور عبد الوهاب المسيري

الناشر: دار الحسام (القاهرة، ص1، 1996) (194ص).

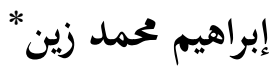

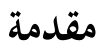

يحتل كتاب أسرار العقل الصهيوني موقفاً متميزاً في نسق مؤلفات الدكتور عبد الوهاب المسيري عن

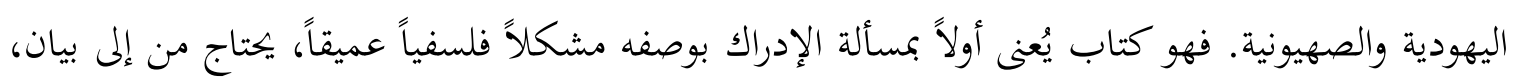

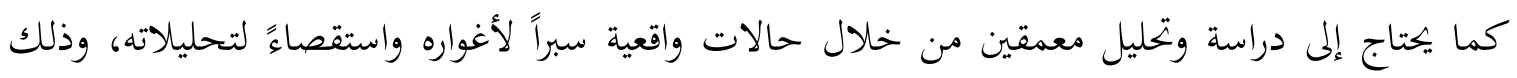

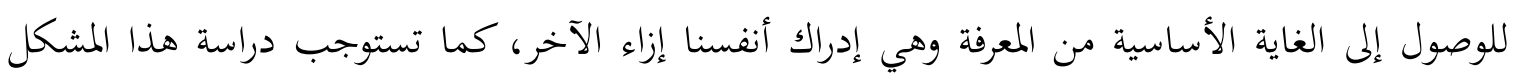

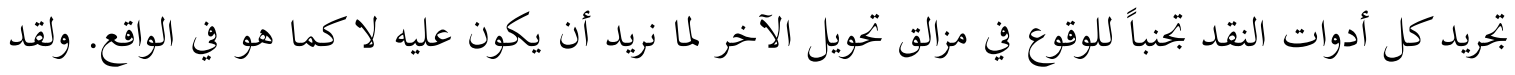
أتاحت الصهيونية بوصفها شبكة من العلاقات الإدراكية حالة مثالية لفهم الصلة بين الذات والآخر، ولفهم مآلات الصلة بين الإدراك والسلوك كما بتلت في الحركة الصهيونية. وبهذا فالكتاب محاولة عميقة للتنظير؛

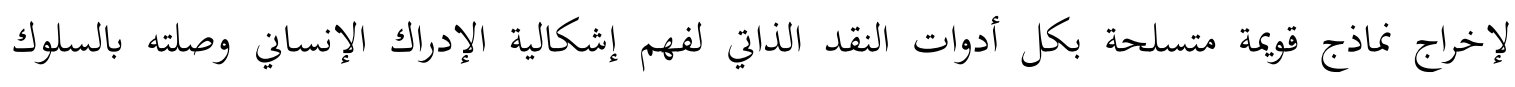
البشري. وقد سعى المؤلف لتطبيق ذلك الإطار التحليلي على حالة الحركة الصهيونية.

وإذا كانت المؤلفات السابقة للدكتور المسيري قد سعت -على مدى ربع قرن من الزمان- إلى رصد

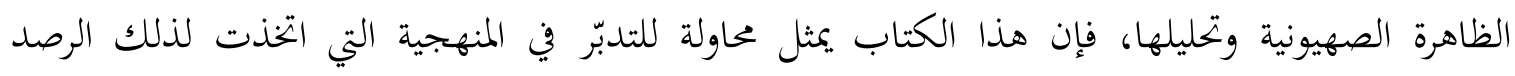

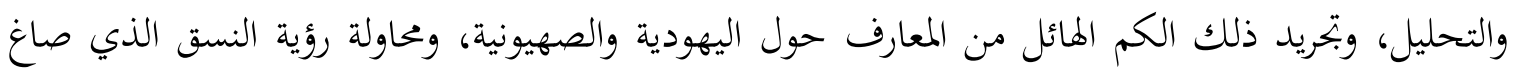
نسيج تلك المعارف. فالكتاب إذن خلاصة فريدة للمنهج الذي اختطه الدكتور المسيري خلال رحلته السابقة في فهم الحركة الصهيونية وربيتها الدولة العبرية.

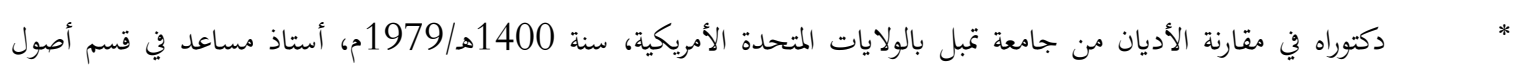
الدين والفلسفة ومقارنة الأديان بالبامعة الإسلامية العالمية باليزيا. 
وهنا تأتي أهمية هذا الكتاب، إذ إنه محاولة واعية لفهم الآخر، ونقد الذات في فهمها للآخر، ثم الارتقاء إلى مرحلة الوعي للنماذج الإدراكية وموقعها في شبكة الإدراك، وصلته بالسلوك الإنساني. فهو يؤرخ لتلك الرحلة المضنية والشائكة لفهم العقل الصهيوني بعقل يتخذ الإسلام هادياً وموجهاً، ولا يفتعل عداءاً عرقياً غايته إثارة النعرات التي تسلب الآخر إمكانية وجوده على وجهه الحقيقي، ومن ثم تقع في براثن الذاتي

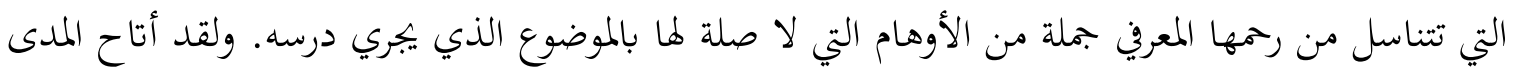
الزمني الذي طور فيه الدكتور المسيري معرفته بحقل الدراسات اليهودية والصهيونية نضجاً ووعياً لمخاطر الانزلاق في الذاتية وتحويل الآخر إلى جملة من الأوهام التي لا صلة لها بمعرفة الآخر معرفة أكمل وأصوب. فهذا السفر يؤرخ لتعقيدات الوعي للآخر، وهمّ التعبير عن ذلك الوعي كما تحلى عبر عشرات الأمثلة والنماذج التي احتواها؛ ويعبر -من جهة أخرى- عن مدى النضج الذي بلغه العقل العربي الإسلامي في التعبير بصدق ووعي عن الآخر المخالف والمساير لنا في العقيدة والوجود.

القضايا الرئيسة للكتاب

يتكون هذا السفر القيم من مقدمة وأربعة فصول. أما المقدمة فقد وصفها المؤلف بأهما تُعنى بالإدراك والسلوك والتبعية الإدراكية، ولكنها علاوة على ذلك تمثل الإطار النظري الذي اتخذه المؤلف لمعالجة قضايا الصراع العربي الإسرائيلي وبعض الأمور المتصلة بذلك الصراع. فالموضوع الأساسي لهذا السفر هو إذن قضية الإدراك بوصفه مسألة نظرية بالغة التعقيد؛ وقد اتخذت مسألة الصراع العربي الإسرائيلي وجملة الموضوعات المتصلة به حالة متعينة لفهم تلك المسألة النظرية.

يحاول الكاتب أن يقدم فهماً للإدراك الإنساني يتجاوز الفهم المادي، الذي يُحيل قضية فهم الإدراك الإنساني إلى جملة من الاختزالات المادية المخلة التي تعد العقل صفحة بيضاء تتراكم عليها معطيات العالم الخارجي المادية، أو ترى أن السلوك الإنساني رهين بأفعال وردود أفعال مشروطة تتحكم فيها في غاية الأمر البيئة الخارجية. ويؤكد الكاتب أهمية النظر إلى العقل البشري بوصفه عقلاً مبدعاً له مقدرة توليدية فائقة، وهو كذلك مستودع لكثير من الخبرات والمنظومات، التي تتجلى في جملة من القيم الخلقية والتشكيلات 
الرمزية التي تتقاطع وتتداخل لتكوِّن في فاية الأمر النماذج المعرفية الإدراكية التي تحدد للإنسان مجال الرؤية والفهم للأشياء والأحياء من حوله، وتمنحه خصوصيته التي تتعالى على القوانين المادية البحتة؛ فتجريد الإنسان من ذلك خطأ فادح يترتب عليه خلل عميق في فهم مسألة الإدراك الإنساني. ولذلك يرى الكاتب أننا لا يمكن أن ندرس الظاهرة الإنسانية كما ندرس الظواهر الطبيعة. فالعملية الإدراكية لدى الإنسان تتم عن طريق النموذج المعريف الذي هو بمنزلة الأداة التي تستخدم لرؤية الواقع المادي الخارجي، وتضيق وتتسع رؤيتنا لذلك الواقع المادي الخارجي بحسب النموذج الذي نتخذه. وهذا لا يعني بأيٍّ حال من الأحوال أن الواقع المادي الخارجي لا وجود له، ولكن يعني أن ما نتمثله من ذلك الواقع المادي خاضع للنموذج المعريف الذي نستبطنه، فضلاً عن أن الواقع المادي له أثر في السلوك البشري، وله وجوده الموضوعي المنفصل عن

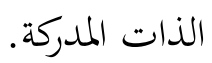

على أن الأمر الذي يُراد تأكيده هنا بوجه خاص هو محورية النموذج المعرفي في العملية الإدراكية؛ وكذلك فإنَّهَ لا توجد حتمية قسرية بين السلوك الإنساني والإدراك، وإنما السلوك الإنساني أم مركب ومعقد غاية التعقيد، تحدده عناصر متداخلة من بينها إدراك الإنسان لواقعه. وعليه فإن الصلة بين الإدراك والسلوك علاقة احتمالية؛ أي أن الإنسان بما حباه الله من نعمة العقل والرشد والقدرة على الفعل المستقل يستطيع بتحاوز مزالق الإدراك المتحيز إلى رحابة الإدراك الإنساني المتميز، الذي يتحلى بأدب الحوار مع الآخر، وإفساح مساحة لوجوده وفعله كما هو لا كما نريده أن يكون، أما إذا أخلد الآخر إلى إدراك ضيق (فاشي) عنصري - كما هو الحال عند الصهيونية- ممسك بمدفع رشاش فالسبيل الوحيد للحوار معه هو ما أسماه الكاتب "ب الحوار المسلح" (ص81).

والغفلة عن أهمية النموذج المعريف في العملية الإدراكية للآخر توقع في خلط خطير، وخير مثال لذلك نظرة الخطاب العربي السياسي المعاصر للصهيونية والحضارة الغربية وللذات العربية نفسها. فحينما نُسقط النموذج المعري وننظر إلى الدولة الصهيونية من زاوية العتاد المادي والعسكري أو النظام السياسي القائم فيها، ونقارنه بالنظم الديمقراطية الغربية، فإننا نقوم بتطبيع النظام السياسي الإسرائيلي والدولة الصهيونية، ولذلك لا نستطيع أن نفسر عمل الوكالة اليهودية، ولا قانون العودة، ولا ضخامة الدعم المادي والمعنوي الذي يقدمه 
الغرب للدولة الصهيونية. كل ذلك يجعل نموذجنا التفسيري عاجزاً عن فهم الطبيعة الإحلالية للدولة الصهيونية والبعد الوظيفي الذي تقوم به بتحاه المشروع الإمبريالي الغربي في المنطقة العربية.

ثم ينتقل الكاتب إلى قضية بالغة الخطورة متصلة بمسألة الإدراك، وهي قضية التبعية متمثلة فيما أسماه "بإمبريالية المقولات"، وقد بحلى ذلك في الخطاب السياسي العربي من خلال المصطلحات السائدة فيه مثل: "المسألة الشرقية"، و"رجل أوربا المريض"، دون وعي التحيز الإمبريالي الكامن وراء تلك المصطلحات، ومثل ذلك مصطلح "الصهيونية العالمية" التي ليست سوى "الصهيونية الغربية"، فالعالم أوسع من الغرب. وكذلك وصفُ بعضِهم الصهاينة الأوائل "بالرواد" جرياً وراء الترجمة الحرفية، بينما هم في الحقيقة مغتصبون لأرضنا. ويستطرد المؤلف في تحليل معنى "معاداة السامية"، و"الانتفاضة"، ويربط كل ذلك بمقولته الأساسية في شأن الإدراك والنموذج المعرفي وعلاقته بالسلوك الإنساني، ثم ينتقل أخيراً إلى بيان أهمية الاستعارة والصورة في

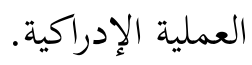

وتُعنى الفصول الأربعة بمحاولة تقديم نموذج محدد للموقف المعرفي الذي التخذه المؤلف في مقدمة الكتاب، ذلك الموقف الذي اعتبره الهمّ الأساسي الذي دفعه للتأليف في هذا البمال. أما الفصل الأول فيركز على مسألة الإدراك الصهيوني للعرب، وذلك أمر منطقي وتاريخي، ويتلوه الفصل الثاني الذي يتصل به كذلك اتصالاً منطقياً وتاريخياً، ليخصص لفهم الإدراك الإسرائيلي للعرب، ثم يأتي الفصل الثالث -الذي يجتاج لمسوغ غير التسلسل المنطقي والتاريخي حتى يربط بالفصول السابقة-ليعالج مسألة الإدراك الغربي لليهود، ثم أخيراً يخصص المؤلف الفصل الرابع لتفكيك الإدراك الصهيوي محدداً جملة من المواقع المعرفية والفكرية في شأن قضية الإدراك والنموذج المعرفي.

لقد عالج المؤلف معالجة دقيقة مستويات الإدراك الصهيوني للعربي، وبيَّن الصلة بين تلك المستويات التي تبدأ بالعربي المتخلّف لتنتهي بالعربي الغائب، ثم استطرد في بيان طرافة إدراك العربي بوصفه يهودياً واليهودي بوصفه عربياً من قبل تلك المنظومة الصهيونية في الإدراك وتمثيل الآخر. ثم انتقل من بعد ذلك لبيان مستويات الاستجابة الصهيونية للعربي الحقيقي، ملحقاً بذلك كيفية استجابة العرب للوجود الصهيوني على أرضهم وسبل تعاملهم مع الدعاية والرؤية الصهيونيتين. 
بيّن المؤلف السياق التاريخي والفكري الذي تأسست فيه وانطلقت منه الفكرة الصهيونية، ورّز على أهمية النظر للفكرة الصهيونية على أساس أها التي طوَّرت فيها هذه الحضارة ملامح مشروعها الاستعماري الإمبريالي وغزت على أساسه العالم بدعوى (عبء الرجل الأبيض التحضيري). إذاً فالفكرة الصهيونية قد انبثقت من الجذور الفكرية ذاتا التي سوغت الاستعمار والعنصرية وباركت الظاهرة الإمبريالية، وعليه فإن الصهيونية في جوهرها رؤية عنصرية تستلهم الرؤية الغربية العنصرية التي سعت إلى جعل الذات القومية مصدراً للقيم، بوصفها المطلق الوحيد الذي يجب أن يؤمن به الفرد في تلك الجماعة القومية، ومن ثم يُصبح كل ما هو خارج تلك الذات بجردَ أدوات أو وسائل لتحقيق تلك الذات. وحينما يصير ما هو خارج عن الذات عائقاً من عوائق تحقيق ذلك المشروع، فإنه يجب أن يُغيَّب أو يزال. وهذا ما لاحظه الكاتب وحشد له

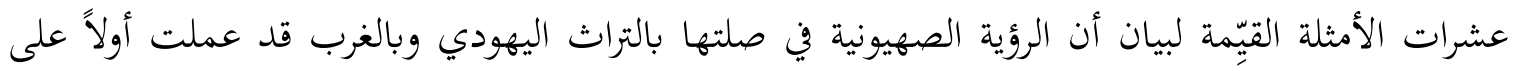
إحلال الرؤية القومية محل ذلك التراث، وقامت بإحلال العقيدة العرقية (الإثنية) الصهيونية مكان العقيدة اليهودية الدينية، وصارت تلك الذات الجديدة هي المطلق الوحيد الذي تُستمد منه القيم ويَسعَى للتحقق في التاريخ. ولما كان ذلك يقتضي تنزيلَ المشروع الصهيوني على أرض فلسطين، اقتضى الأمر ضرورة تغييب مَنْ على تلك الأرض من بشر وإزالته بما يحمل من إرث ثقافي وحضاري. وعليه، ومثلما أن الفكرة الصهيونية قد حلت مكان العقيدة الدينية وصارت الذات المطلقة المفردة، فهي كذلك قد سعت للتحقق التاريخي بتغييب العربي وإزالته من منظومتها الإدراكية حتى يستبب الأمر لتلك المنظومة وتصل إلى غاياتا المنطقية.

ويمكننا القول مع المؤلف: إن المنظومة الإدراكية الصهيونية في لحظة نادرة تُدرك العربي كما هو، ثم تسعى لتغييبه تدريجياً عبر تدابير إدراكية تنحو إلى تجريد وجوده من النظر إليه إنساناً إلى النظر إليه معنيً مجرداً يمنح الفكرة الصهيونية تحققها التاريخي التام على "أرض إسرائيل". وقد سعى المؤلف إلى إثبات هذه الاستراتيجية الإدراكية في النظر إلى الآخر من خلال المؤلفات والتصريحات التي تمثل جماع الرؤية الصهيونية للعربي. وقد أنتجت تلك الرؤية جملة من المقالات الأساسية مثل: "العربي المتخلف"، و"العربي ممثلاً للأغيار"، و"العربي الهامشي"، و "العربي الغائب"، إلخ. وقد بيّن المؤلف التدرج الذي يجعل كل مقولة من هذه المقولات مرحلة في تحقيق الغرض الأساس من هذه الرؤية الصهيونية، وهو النفي التام للوجود العربي. وقد نجح المؤلف في حشد شواهد قِّمة تمثل شخصيات أسهمت بنصيب وافر في تشكيل الرؤية الصهيونية. 
ولئن كانت المقولات التي ذكرها المؤلف مستقاة أساساً من المشروع الاستعماري الإمبريالي الذي أعطى الحركة الصهيونية مسوغ وجودها، فإن تلك الحركة قد خطت خطوات واسعة -وأحياناً متناقضة- في الاستراتيجية التي نفّذت بها تلك المقولات. فمثلاً إن النظر إلى العربي بوصفه "كائنا متخلفاً" قد انبنى على غرار نظرة الحضارة الغربية إبان مشروعها الاستعماري إلى الآخر لتسويع معنى مسؤولية الرجل الأبيض التحضرية! لكن الرؤية الصهيونية - بسبب من نزوعها إلى تغييب العربي - لم تتخذ التدابير اللازمة لانتشال العربي من وهدة تخلفه ورفعه إلى مصاف الحضارة والتقدم، بل اتخذت كل الأساليب التي تبقي العربي على تخلفه، وفي بعض الأحيان سعت سعياً واعياً لتكريس ذلك التخلف. وهذا التناقض في الرؤية الصهيونية ناجم عن ازدواجية النظرة الصهيونية التي تستخدم المقولات السابقة لا للتعبير عن واقع بعينه، ولكن لتسويغ المشروع الصهيوني الذي يسعى إلى غاية فائية لا صلة لها بتلك المقولات، إلا إذا كانت تلك المقولات الإدراكية تخدم الغرض الأساسي وهو نفي العربي وتغييبه، أيّ أن تلك المقولات لا يمكن أن تفهم بمعزل عن الهدف الاستراتيجي.

وقد حاول المؤلف تحليل نماذج للاستجابة الصهيونية للعربي الحقيقي: منها استجابة المفكر الصهيوني الروسي الذي اتخذ لنفسه اسماً سمتعاراً (آحاد هعام)، والذي احتج منذ البداية على طريقة فهم المشروع الصهيوني للعربي الفلسطيني، وماولة تغييه. وقد بلغ إدراكه الذروة حينما بيَّن التلازم الحتمي بين حلم صهيون في العودة إلى أرض الميعاد ودماء الفلسطينيين التي ستكون ثمناً لتلك العودة، فندّد بهذه العودة الوحشية التي لا تتسق مع معاني الأمة الإسرائيلية التي يفترض أن تكون قدوة لأمم الأرض؛ أما أن تدنس أرض إسرائيل -حسب زعمه- بدماء الأبرياء ليتحقق حلم العودة، فذاك أمر لا يحتمله معنى العودة إلى أرض إسرائيل التي تحقق معنى المسيح المخلص الذي وعد به الأنبياء في بشاراتم. ومثل أحاد هعام يرى اسحق اينشتان وكذلك بن جوريون وموشيه شاريت: العربي الحقيقي خارج مدى التزييف الذي وقعت فيه الرؤية 
ولكن يبقى السؤال الجوهري: براُ لَمْ تصر هذه الرؤية للعربي الحقيقي جزءاً حياً في تشكيل الرؤية

الصهيونية؟

يعترف المؤلف بأن الإجابة عن هذا السؤال تقتضي تطوير معرفة لفهم الصلة بين الأفكار والواقع، منبهاً إلى أن العلاقة بين الإدراك والسلوك علاقة احتمالية ومؤكداً أهمية الوعي أن التحليل الذي يقدمه إنما هو محاولة ذهنية - في المقام الأول-لتفسير واقع معين، وأن إدراك الصهاينة للعربي الحقيقي قد يترجم نفسه إلى جملة من الأفعال المتناقضة حسب موقع المدرك الذي تحدده جملة من العوامل التاريخية والسياسية والنفسية والعصبية. وقد حاول المؤلف تقسيم الاستجابات الصهيونية إزاء العربي الحقيقي ثلاثة أنماط:

أولها: أولئك الذين أدركوا أول وهلة الجرم الكامن في المشروع الصهيوني في عملية تغييب العربي، فتنكروا للرؤية الصهيونية -ومن هؤلاء كثيرون من حزب بوعالي صهيون (عمال صهيون) -فرجعوا إلى الاتحاد السوفيتي وشاركوا في الثورة البلشفية. ويبدو أن هؤلاء قلّة نظراً لندرة الإشارات إليهم في المدونات الصهيونية. ويرى الكاتب أهمية استقصاء حالات هؤلاء والتعرف عليهم وإعادة كتابة تاريخ الصهيونية الذي سعى لتغييبهم.

ثانيها: أولئك الصهاينة الذين أدركوا العربي على حقيقته، وحاولوا إعادة صياغة المشروع الصهيوني ليضع في الحسبان ذلك العربي لا ليقوم بتغييبه، ومن هؤلاء أبنشتاين وآرثر وروين، وربما آحاد هعام. لكن هذا "الوعي التعس" - كما تقول عبارة هيجل التي نستعيرها هنا- لم يسعف هؤلاء في أن يكون لمم تأثير في المشروع الصهيوني، بل كان ذلك الوعي سبباً في تمميشهم وإقصائهم عن مراكز صنع القرار.

ثالثها: أولئك الصهاينة الذين أدى بهم إدراكهم للعربي على حقيقته إلى أن يتخذوا مواقف أكثر شراسة، وأن يدفعوا بالمشروع الصهيوني نو غاياته المنطقية، وهي تغييب العربي، وهذا النمط من الاستجابة الصهيونية يمثل السواد الأعظم. ثم يحاول الكاتب بيان الأسباب التي جعلت هذا النموذج أكثر شيوعاً وسط الصهاينة المتطرفين، أو قل الواقعيين، هم أفضل من أدرك وضع العربي الحقيقي في المشروع الصهيوني. فالشراسة التي يتعاملون بها معه قائمة على فهم خطورة ذلك العربي على المشروع الصهيوني وعلاقة القوى التي يفترضها أو يبني عليها. وخير مثال لذلك أطروحات فلاديمير جابوتنسكي فيما أسماه بالحائط الحديدي 
بين الصهاينة والعرب، أو السلام الذي يفرض على العرب بالقوة كما بشر به بن غوريون، وكذا الحال عند شاريت ووايزمان؛ فكل هؤلاء في فاية الأمر، وبسبب الفكرانية (الأيديولوجية) الصهيونية وعلاقات القوى التي تطرحها، يسعون فقط "لسلام المقابر" مع العرب.

وإزاء هذه الرؤية الصهيونية وعلاقات القوى التي تفترضها في فهم مشروعها وفي تحقيقها، قام الوعي العربي والاستجابة العربية في وقت مبكر ممثلين في نجيب عازوري "الفلسطيني العربي المسيحي" (صك2) كما وصفه الكاتب- الذي رأى أن الصراع سيستمر إلى أن يسود طرف على آخر. وعلى الرغم من النزعة التشاؤمية الواضحة في العبارة السابقة، إلا أن الكاتب يرى أن هذا القول أكثر واقعية في فهم المضامين الأساسية للمشروع الصهيوني، التي مهما زينت بعبارات معتدلة ودعوات اعتذارية، إلا أن المنطق الأساسي الذي يحكمها يُفضي إلى صراع، الغرض منه تغييب العربي. لقد فهم كلا الطرفين -العربي الحقيقي وأصحاب المشروع الصهيوني- موقف كل منهما إزاء الآخر، ولم يعد الأمر مجرد نوايا حسنة، وإنما قضى المشروع الصهيوني بأن السلام الأمثل للعربي هو "سلام القبور" (صنهري)".

ينتقل المؤلف بعد ذلك إلى تطبيق المنهجية ذاتا في فهم الإدراك الإسرائيلي للعرب ولمشروع الدولة الفلسطينية والانتفاضة. فيرى أن الوجدان الإسرائيلي لا يزال حبيساً للإدراك الصهيوني الغربي بكل تحيزاته، بل إن ذلك قد انعكس سلباً على يهود المنطقة العربية الذين تنظر إليهم الدولة الصهيونية على أفم عنصر من عناصر التخلف العام في الكيان الصهيوني. ومن ثم فالدولة الإسرائيلية لم تتخلص من الرؤية العنصرية الغربية التي غذهّا فلسفات القرن التاسع عشر، ووجدت مسوغاتما في المشروع الاستعماري الإمبريالي الذي تُقاخر بالانتماء إليه، وإن أدى ذلك إلى التنكر للتراث اليهودي الثري الذي أنتج في العصور الوسطى ضمن

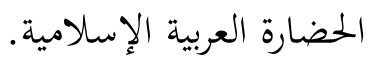

ومرة أخرى يحلل الكاتب وفق نموذجه التفسيري موقع العربي في المنظومة الإدراكية الإسرائيلية ويششد عشرات الأمثلة ذات الدلالة العميقة في فهم العقل الإسرائيلي في صلته بالآخر. فالعربي المتخلف، والعربي مثثلاً للأغيار، والعربي الهامشي، والعربي الغائب، ثم أخيراً عملية الإسقاط في رؤية العربي يهودياً، كل هذه الصور من الإدراك تتكرر مرة أخرى لتعطي المشروع الصهيوني في الدولة الإسرائيلية مسوغاً معرفياً وبتعل 
الاستجابات الصهيونية الثلاثة السابقة محدداً للفاعلية داخل الكيان الصهيوني في الثلاث: أولها: أن يتخلى الإسرائيلي عن صهيونيته، وثانيها: أن يعدل من صهيونيته فيتحول إلى شخصية هامشية، وثالثها: أن يتمسك بصهيونيته ويزداد ضراوة نظراً لتزايد إحساسه بالخطر المحدق. وهذه النماذج الثلاثة تتكرر في الدولة الإسرائيلية والإدراك الإسرائيلي، وإزاءها ينعقد لواء الفاعلية داخل الدولة. ومثلما كان النموذج الثالث حامل لواء الرؤية الصهيونية، يبدو كذلك جلياً أنه هو الذي يمنح تلك الدولة مسوغ وجودها. وعلى ذلك فإن الاعتدال والتطرف الإسرائيلين هما نتاج بنية إدراكية واحدة قائمة على قصور إدراكي، يخلق حالة من الصراع مع الآخر، ذلك الذي ولّد حروباً لن تنتهي إلا باستسلام أحد الطرفين للآخر.

ويطبق المؤلف الأدوات التحليلية نفسها في فهم الإدراك الإسرائيلي للعربي الحقيقي على قضيتين

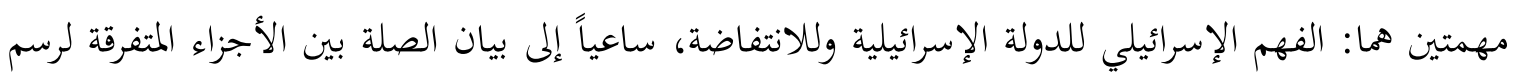
صورة كلية متوازنة عن هاتين القضيتين في فهم الصراع العربي الإسرائيلي. وقد استطاع بذلك تعرية الوهم الذي يساور بعض العرب -ساسة ومثقفين-في مسألة الاعتدال الصهيوني الذي أبان أنه إنما يمثل مرحلة في المشروع الصهيوني الساعي لتغييب العربي؛ مثلما كان خيار "الأرض في مقابل السلام" خياراً إجرائياً وليس تنازلاً هائياً عن "أرض إسرائيل" لأن القدرة الذاتية للدولة الإسرائيلية وموازين القوى الدولية لا تسمح بغير ذلك، فيتم التنازل عن الأرض وفق مفهوم لا يناقض المنظومة الإدراكية الإسرائيلية الصهيونية التي تسعى لتغييب العربي. وقد أكد المؤلف أهمية النظر إلى المنظومة الإدراكية الصهيونية الإسرائيلية، لا بحسباها منظومة عنصرية استعمارية فحسب، ولكن بالنظر إلى ما تنطوي عليه من نزعة إحلالية.

وحينما يُحلل المسيري الموقف الإدراكي الإسرائيلي بتحاه الانتفاضة الفلسطينية يبين أهمية الاعتماد على نظر واسعة تتخطى ثنائية الاعتدال والتشدد (الحمائم والصقور) لأن هناك "صوراً إدراكية أخرى"، ويقدم أمثلة حية وطريقة تساعدنا على فهم التعقيد في ظاهرة الإدراك الإسرائيلي، ويجعلنا أكثر قدرة على الفهم والتفسير للمنظومة الإدراكية الإسرائيلية تجاه الانتفاضة.

وبعد ذلك ينتقل المؤلف إلى مسألة أخرى ذات أهمية، وهي فهم الإدراك الغربي لليهود، وكيف أن ذلك الإدراك الغربي لليهود قد صاغ القيم التي قامت على أساسها الدولة الصهيونية فيما بعد. فوجود 
اليهودي الذي يقبله الغربي هو دائماً ذلك الآخر الذي يؤدي وظيفة للمجتمع الغربي، ذلك الآخر الذي يتحول أداة، والذي يصبح مغزى وجودِه مرتبطاً بأداء تلك الوظيفة. ومن بعد صارت الدولة الإسرائيلية مجسدة لتلك المعاني التي تطورت عبر تاريخ التعامل بين الغرب واليهود، فبشرت الحركة الصهيونية بدولة وظيفية لليهود. وقد صارت الدولة العبرية فيما بعد تعيد إنتاج مسوغات وجودها وتأكيدها بالوظيفة التي تؤديها للغرب في المنطقة العربية الإسلامية.

حشد المؤلف جملة من النصوص المختارة بدقة للتعبير عن معنى تحوّل اليهودي عبر تاريخه في الغرب إلى شخص يؤدي وظيفة ما. وإذا كان محتوى تلك الوظيفة قابلاً للتغير من مرحلة إلى أخرى، إلا أن السمة الأساسية التي يسوغ بها اليهودي أو الجماعة اليهودية وجودهما في المجتمع الغربي هي مدى أدائهم لتلك الوظيفة التي يحتاج الغرب إليها، فاليهود قد ألفوا ذلك في صلتهم بالغرب، وانتقل هذا المنطق ذاته في التسويغ ليصير تعبيراً عن مغزى وجود الدولة الصهيونية. وهذا التحول حدث في محتوى العمل الوظيفي من كون الجماعة اليهودية تمثل "الشعب الشاهد" في الرؤية المسيحية الكاثوليكية لوجودها المتدني قياساً إلى عظمة الكنيسة، إلى كوها جماعة وظيفية تؤدي عملاً نافعاً للمجتمع الأوربي الحديث الذي سيطرت عليه قيم النفعية والمادية والقوة، فصارت الجماعة اليهودية تقوم بوظائف نفعية تتناسب مع حاجة المجتمع الأوربي. وحينما هضت الدعوة للدولة الصهيونية استُخدِم المنطقُ ذاته لتسويغها، وكذلك عندما ظهرت الدولة الصهيونية إلى حيّز الوجود صارت تعبّر عن هذا المعنى في صلتها بالغرب؛ فهي بذلك دولة قد تمت "حوسلتها" -حسب عبارة الدكتور المسيري - (أي تحويلها إلى وسيلة) لصالح الدولة أو الدول الإمبريالية الراعية لما.

وقد استخدم المسيري جملة من الاستعارات القيمة والذكية في التعبير عن موقع اليهودي والجماعة اليهودية، ثم من بعد الدولة الصهيونية، في صلتها بالغرب، وقد استدعى في تلك الاستعارات معاني تاريخية ذات مغزى عميق يتكرر في التاريخ البشري، ويُ2يد كثيراً في فهم صلة الذات بالآخر، حينما يُصيب المنظومة الإدراكية كثير من التحيّز ضد الآخر.

وبعد أن يبين المؤلف تحول اليهودي إلى عنصر نافع في الحضارة الغربية، ونظر تلك الحضارة إليه، ونظره إلى نفسه على أنه أداة نافعة يحقق وظيفة لا يؤديها سواه، وبين كيفية انتقال ذلك إلى الدولة 
الإسرائيلية نفسها التي صارت دولة وظيفية ترعى أهداف المشروع الإمبريالي في المنطقة، حاول ربط ذلك كله بمفهوم آخر له دلالة خطيرة وهو "اليهودي كمسلم في أفران الغاز"؛ وخطورة هذا المفهوم تنبع من بتسيده لأبعاد عميقة في نظرة الغربي للمسلم "موزلمان."

وقد حاولت الكتابات الإسرائيلية تغطية تلك المنطقة المظلمة في الإدراك الغربي بتحه المسلم بافتراض تبريرات اعتذارية، مما دعا الدكتور المسيري إلى الكشف عن هذه الازدواجية التي يقوم بها العقل الصهيوني الإسرائيلي في صلته بالعربي الحقيقي، فهو من جهة ضحية للإدراك الغربي حينما قبل أن يكون أداة نافعة، وحينما وقع في حالة تميش مزدوج، إذ نظر إليه العقل النازي الغربي "كمسلم في أفران الغاز"، وكذلك عندما أعاد إنتاج معنى الحكم الذاتي بالمفهوم النازي في أطروحاته للحكم الذاتي الفلسطيني هي أن يكون صورة أخرى "لجيتو وارسو" أو مستوطنة "تيريس ينشتات" النموذجية التي أنشئت في عام 1941 لتجميع اليهود من وسط أوربة وغربها في تشيكوسلوفاكيا. فما فعلته النازية -مثثلة للحضارة الغربية-باليهود يعاد إنتاجه مرة أخرى في فلسطين. ولعل النازية في صلتها باليهود لم تخرج كثيراً عن النمط المرسوم لعلاقة الحضارة الغربية بالآخر، فما فُعل باليهود قد سبق فعله في أدغال أفريقيا وسهوب أمريكا بالسكان الوطنيين الأفارقة والهنود الحمر؛ والجديد في الأمر هو أن ألمانيا النازية لم يكن لها مستعمرات فجرت فعلتُها النكراء في أوروبا ذاتما.

ثم ينتقل المؤلف إلى تحليل مسألة الإدراك الغربي والصهيوني لحروب الفرنجة (الصليبين) ملاحظاً عمق التشابه بين المشروع الفرنجي والمشروع الصهيوني الإسرائيلي في المنطقة. ولعل نقطة التشابه الأساسية أن كلاّ من المشروعين استهدف فلسطين، فضلاً عن أن كليهما قد اعتمد على قوى غربية في البقاء، ثم إن الكيان الاستيطاني الذي نشأ نتيجة لذلك المشروع قد مثل حالة بجتمع "مشتول"، كما أن الدولة التي أقامها أخذت شكل "الدولة الجيتو"، أو "الدولة القلعة".

وعلى الرغم من سعي كلا المشروعين للاحتفاظ بكوية متجانسة، إلا أن المادة السكانية دائماً ما تظهر حالة الشتات الذي يشقه. ولقد احتفظت مما لك بالوحدة النسبية التي انتظمت أوربة في ذلك الوقت. وعلى الرغم من استخدام الرموز الدينية في كلا المشروعين، إلا أن تلك الرموز قد أُفرغتت من محتواها الديني 
وأعيدت صياغتها وفق معان قومية و "إثنية" خخالفة للأخلاق المسيحية واليهودية الحقة، وهذا ما يفسر سلوكهما إزاء الآخر. وأخيراً، وعلى الرغم من اعتماد المسيري على تحليل أفنيري في كتابه إسرائيل بدون صهيونية لفهم الصلة بين محالك الفرنجة والدولة الصهيونية وكذلك في مقاله "ماذا ستكون النهاية"، إلا أنه قد نجح في وضع مسألة محالك الفرنجة ضمن السياق العام الذي رسمه لفهم المنظومة الإدراكية الصهيونية الإسرائيلية وصلتها بالمنظومة الإدراكية الغربية في فهمهما للعربي المسلم الحقيقي وسلوكهما إزاءه.

إن الفصل الرابع أكثر الفصول تعقيداً وتركيباً، ويقتضي تركيزاً خاصاً لتتبع نسيج خيوطه في تفكيك الإدراك الصهيوني وإعادة تركيبه لتبيّن منهج التحليل (النماذجي) سبيلاً لفهم الآخر ورصد الواقع من خلال نماذج معرفية. ويمكن القول هنا: إن هذا الكتاب هو في المقام الأول عن الإدراك البشري.

وقد حاول المسيري بيان كيفية قيام العقل الصهيوني باستخدام وقائع وأحداث وأساطير حول مسألة المعاداة للسامية لإعادة صياغتها في نسق الإدراك الصهيوني، وإعطاء تلك الوقائع معنى صهيونياً يصب، في هاية الأمر، في خدمة الأهداف الصهيونية؛ وقد كانت الإستراتيجية الأساسية التي استخدمت في ذلك هي اجتزاء تلك الوقائع من سياقها التاريخي، تم تجريدها من أي مضمون اجتماعي إنساني، وإعادة إنتاجها وفق مضامين صهيونية تندرج في سياق الإدراك الصهيوني، وقد نجح المؤلف في تفكيك الوقائع الثلاث التي اختارها -بموضوعية ودقة-ثم في إعادة فهمها وفق سياقها التاريخي الاجتماعي الإنساني العام، وبيان وجه الحقيقة التي سعى العقل الصهيوني لإخفائها، لا عن طريق تبديل الوقائع في ذاتا، ولكن عن طريق إعطائها معنيً صهيونياً. وقد برع المؤلف في استقصاء حالات ثلاث قام بتفكيكها وفق منهجية صارمة تظهر عمق الوعي الذي توفر عليه في فهم العقل الصهيوني. ولعل تحليله لأسطورة "قمة الدم" و "واقعة الفرد دريفوس" وواقعة "محاكمة ليو فرانك" (وهي الوقائع التي أشرنا إليها قبل قليل) تبين لنا جانباً مهماً في المنظومة الإدراكية الصهيونية في محاولتها إعادة إنتاج تلك الوقائع لخدمة أهدافها العليا.

وقد أثار المؤلف موضوعاً شائكاً حينما حاول بيان صلة بين الحركة الصهيونية والرومانسية، ولكنه بقدرة فائقة على التحليل بيّن السمات الرومانسية للمشروع الصهيوني تبييناً يمكننا من فهم الأبعاد الإدراكية العميقة للرومانسية المتعلقة في الأصل بالعودة إلى عالم يسهل فيه العثور على "المطلق" وعلى "الثبات" الذين 
يطلقان طاقات الخيال والإبداع ضمن عالم يتسم بالوحدة العضوية. فكذلك الصهيونية في عمقها تكرس أفكاراً مطلقة ثابتة مثل "الشعب المختار" و"الأرض المقدسة"... إلخ. وقد ميز المسيري كذلك بين الصهيونية والرومانسية حتى لا يقع اللبس بسبب الصلة التي عقدها بينهما.

وأخيراً يؤكد المسيري في الجزء الذي أفرده لمناقشة "الإدراك والمقدرة التنبئية للنموذج" أهمية استرداد العامل الإنساني لأي نموذج إدراكي. ثم إنه يقيم علاقة طردية بين إحاطة النموذج بالظاهرة في كل أبعادها وزيادة مقدرته التفسيرية والتنيئية. وهذا الجزء من الفصل يمثل خلاصة الكتاب بأكمله، ويمثل كذلك فهماً حقيقياً للمنظومة الإدراكية الصهيونية الإسرائيلية.

أولاً: يمثل هذا الكتاب مثالاً جيداً وموحياً لموقف المثقف العربي المسلم بتحه قضايا أمته، فهو يتحمل هم البيان دون تريج أو إسفاف، وبإحاطة وشمول وشجاعة. ففي أجواء "سلام المقابر"، يعز علينا أن نجد ذلك المثقف العربي المسلم الذي يدعو لما أسماه "الحوار المسلح: بوصفه الطريق الوحيد لرد الأمر إلى نصابه، ولتعامل مع المنظومة الإدراكية الصهيونية من موقع العربي الحقيقي لا من موقع العربي الغائب، كما هو حال تلك المنظومة في تعاملها مع الآخر.

ثانياً: لقد أثبت المسيري مقدرة عالية على نحت المصطلحات وتوليدها، وابتكار الاستعارات الخلاقة والساخرة في فهم صلتنا بالآخر، وبيان أبعاد المنظومة الإدراكية الصهيونية الإسرائيلية. ولعلّ كثيراً من تلك المفاهيم والاصطلاحات والاستعارات أن تسهم في إثراء ثقافتنا ووعينا بذاتنا وبالآخر.

ثالثاً: لا شك أن هناك جملة من الكتب التي خطها العقل الصهيوني الإسرائيلي في فهم ذاته المعاصرة أو فهم العقول التي أنتجتها الذات اليهودية في تاريخها. وعلى الرغم من عدم دخول المسيري في حوار مع بعض تلك المؤلفات، إلا أن كتابه يُعد فتحاً جديداً في هذا المجال، ولا شك أن له قيمة علمية وفكرية 
ستسهم في فهم الصهيوني الإسرائيلي لنفسه، والبون شاسع بن ما كتبه روفائيل ياتاي عن العقل اليهودي والعقل العربي وأطروحة المسيري في هذا الكتاب.

رابعاً: إن هذا الكتاب قد أكد معنى أن المسيري حجة في فهم الظاهرة الصهيونية الإسرائيلة، وقد كنت أحسب أن الحضارة العربية الإسلامية لن تنتج مؤلفاً فذاً ومغامراً مثل البيرولي مرة أخرى، ولكن بعد قراءة هذا السفر أرى أن ظاهرة البيروني تتكرر وتحمل معها المعاني مجسدة في كتاب فلعيه بأسرار العقل الصهيوني.

أخيراً: على الرغم من القيمة العلمية والفكرية المتميزة لهذا الكتاب، إلى أن دار الحسام قد أخفقت تماماً في مواكبة التقدم والنضج اللذين بلغتهما صناعة الكتاب في مصر من حيث صف مادة الكتاب وإخراجها، وإعداد الغلاف، وكل ما يتبع ذلك من مسائل فنية في هذا الصناعة، ذلك فضلاً عن الأخطاء المطبعية العديدة التي بعضها قد يؤدي إلى تشويه الرسالة التي أراد المؤلف تبليغها. 